\title{
2H-Pyrazol-3-ylamines as precursors for the synthesis of polyfunctionally substituted pyrazolo[1,5-a]pyrimidines
}

\author{
Hany Fakhry Anwar, ${ }^{* a}$ Daisy Hanna Fleita, ${ }^{b}$ Heinz Kolshorn, ${ }^{c}$ Herbert Meier, ${ }^{c}$ and \\ Mohamed Hilmy Elnagdi ${ }^{a}$ \\ ${ }^{a}$ Chemistry Department, Faculty of Science, Cairo University; Giza; A. R. Egypt. \\ ${ }^{b}$ Chemistry Department, American University in Cairo; Egypt \\ ${ }^{c}$ Institute of Organic Chemistry, Johannes Gutenberg-University, Mainz, Germany
}

E-mail: hany.anwar@farmasi.uio.no

\begin{abstract}
Substituted aminopyrazoles (5a-d) were synthesized and reacted with bidentate electrophiles to afford pyrazolo[1,5-a]pyrimidines. The regioorientation of reagents has been determined by $\left({ }^{15} \mathrm{~N},{ }^{1} \mathrm{H}\right) \mathrm{HMBC}$ measurements as well as an X-ray crystal structure determination.
\end{abstract}

Keywords: 7-Aminopyrazolo[1,5-a]pyrimidines, amino-imine tautomerism, HMBC, HMQC experiments

\section{Introduction}

The synthesis and chemistry of pyrazolo[1,5-a]pyrimidines have recently been revived as revealed by the vast number of papers and patents which report routes for the synthesis of different biologically active pyrazolo[1,5-a]pyrimidine derivatives. ${ }^{1-6}$ The recent discovery of Zaleplon (1) as an ideal hypnotic drug has stimulated further interest in the pyrazolo[1,5$a$ ]pyrimidine chemistry. ${ }^{7,8}$ Pyrazolo[1,5-a]pyrimidines are readily obtained via reacting bidentate electrophiles with 3-amino-1H-pyrazoles. ${ }^{9}$ If the reagent is symmetrical or a monocyclic intermediate is isolable, defining the exact structure of the reaction product does not make any significant problem. However, in some cases the only isolable products are the finally formed pyrazolo[1,5-a]pyrimidines. ${ }^{10,11}$ In such cases the identification of the exact regioorientation of the reactants could be only established with certainty by X-ray crystal structure determinations. ${ }^{12}$ In the past ${ }^{9}$ we have described several synthetic approaches to pyrazolo[1,5-a]pyrimidines via reacting $\alpha, \beta$-unsaturated nitriles and esters with 3-amino- $1 H$-pyrazoles. The assigned structures were mainly based on the observed position of amino or carbonyl functions in ${ }^{1} \mathrm{H}$ NMR and IR spectra. Now we report on a more conclusive structure elucidation by applying $\left({ }^{15} \mathrm{~N},{ }^{1} \mathrm{H}\right) \mathrm{HMBC}$ experiments. 


\section{Results and Discussion}

The aminopyrazole 5a, that has been selected as starting material, could be prepared via reacting 2 with hydrazine hydrate in a microwave oven in the presence of acetic acid. On the other hand 5b-d were prepared via coupling $\mathbf{3}$ with aromatic diazonium salts and subsequent refluxing of the so formed arylhydrazones $\mathbf{4 b - d}$ with hydrazine hydrate in ethanolic solution (Scheme 1).<smiles>CCN(C(C)=O)c1cccc(-c2ccnc3c(C#N)cnn23)c1</smiles>
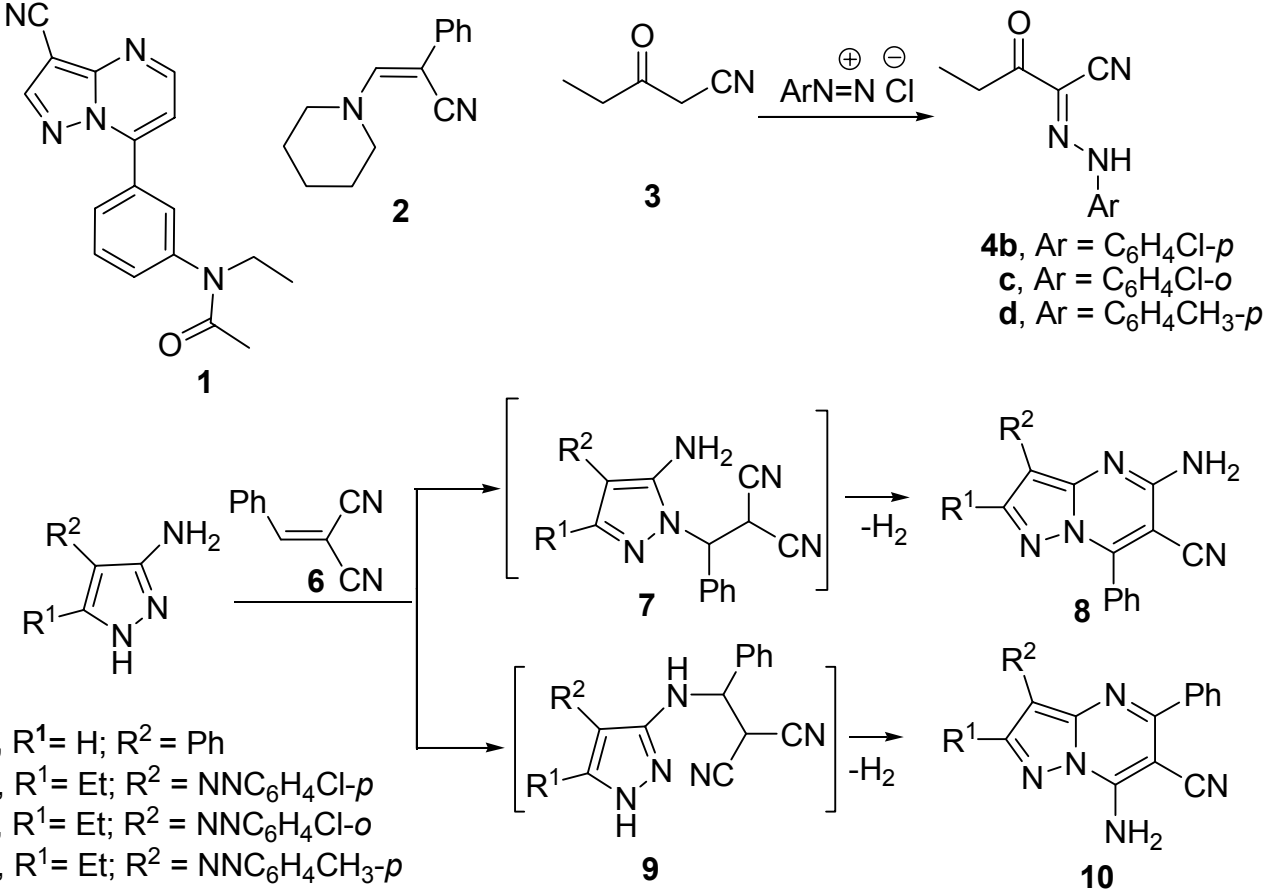

\section{Scheme 1}

Compounds 5a-d reacted with benzylidenemalononitrile $\mathbf{6}$ to yield aminopyrazolo[1,5a]pyrimidines that may be formulated as 8a-d or isomeric 10a-d. Thus if the initial addition involves ring nitrogen atom $\mathrm{N}-2$, as has been assumed earlier by Elnagdi et al. ${ }^{10}$, Michael adduct 7 would be formed. This then cyclizes to yield 8. On the other hand, if the exocyclic amino function reacts with the electrophilic carbon atom of $\mathbf{6 , 9}$ would be formed. Its cyclization and autoxidation would then afford $\mathbf{1 0}$ (Scheme 1). Reacting 5a with $\mathbf{2}$ also afforded products that can be formulated as $\mathbf{1 1}$ or isomeric $\mathbf{1 2}$ (Scheme 2).

Figure 1 shows an ${ }^{15} \mathrm{~N},{ }^{1} \mathrm{H}$ - heteronuclear multiple bond correlation (HMBC) of compound 11 measured in $\mathrm{CD}_{3} \mathrm{SOCD}_{3}$. Crosspeaks for all nitrogen atoms $\left(\mathrm{N}-1, \mathrm{~N}-4, \mathrm{~N}-7 \mathrm{a}\right.$ and $\left.7-\mathrm{NH}_{2}\right)$ and the protons $2-\mathrm{H}, 7-\mathrm{H}$ and $\mathrm{NH}_{2}$ can be observed provided that they are connected by not more than 4 bonds. The size of the coupling constants $J\left({ }^{1} \mathrm{H},{ }^{15} \mathrm{~N}\right)$ corresponds to the sequence $\left|{ }^{1} J\right|>\left.\right|^{2} J \mid$ $\left.\approx\right|^{3} J|>|^{4} J$. N-7a with two ${ }^{3} J$ and one ${ }^{4} J$ coupling gives the largest signal and N-4 with one ${ }^{2} J$ and one ${ }^{4} J$ coupling the smallest. The position of the amino group on C-7 is unambiguously determined by the ${ }^{3} J$ coupling of its protons with the nodal nitrogen atom N-7a. The coupling ${ }^{5} J$ 
$\left(\mathrm{NH}_{2}, \mathrm{~N}-7 \mathrm{a}\right)$ of the alternative structure 12 (bearing the $\mathrm{NH}_{2}$ group on C-5) would not be visible in the spectrum. On the other hand, large crosspeaks for ${ }^{3} J(7-\mathrm{H}, \mathrm{N}-1)$ and ${ }^{2} J(7-\mathrm{H}, \mathrm{N}-7 \mathrm{a})$ should appear in the HMBC spectrum, when the isomer with a $5-\mathrm{NH}_{2}$ group would be present. Thus, structure $\mathbf{1 2}$ can be ruled out.

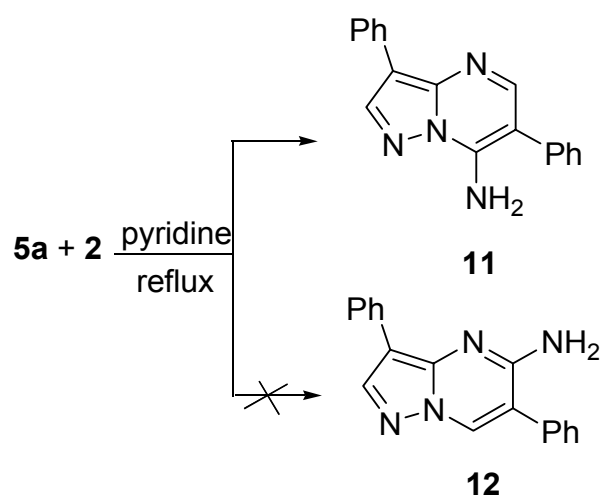

Scheme 2

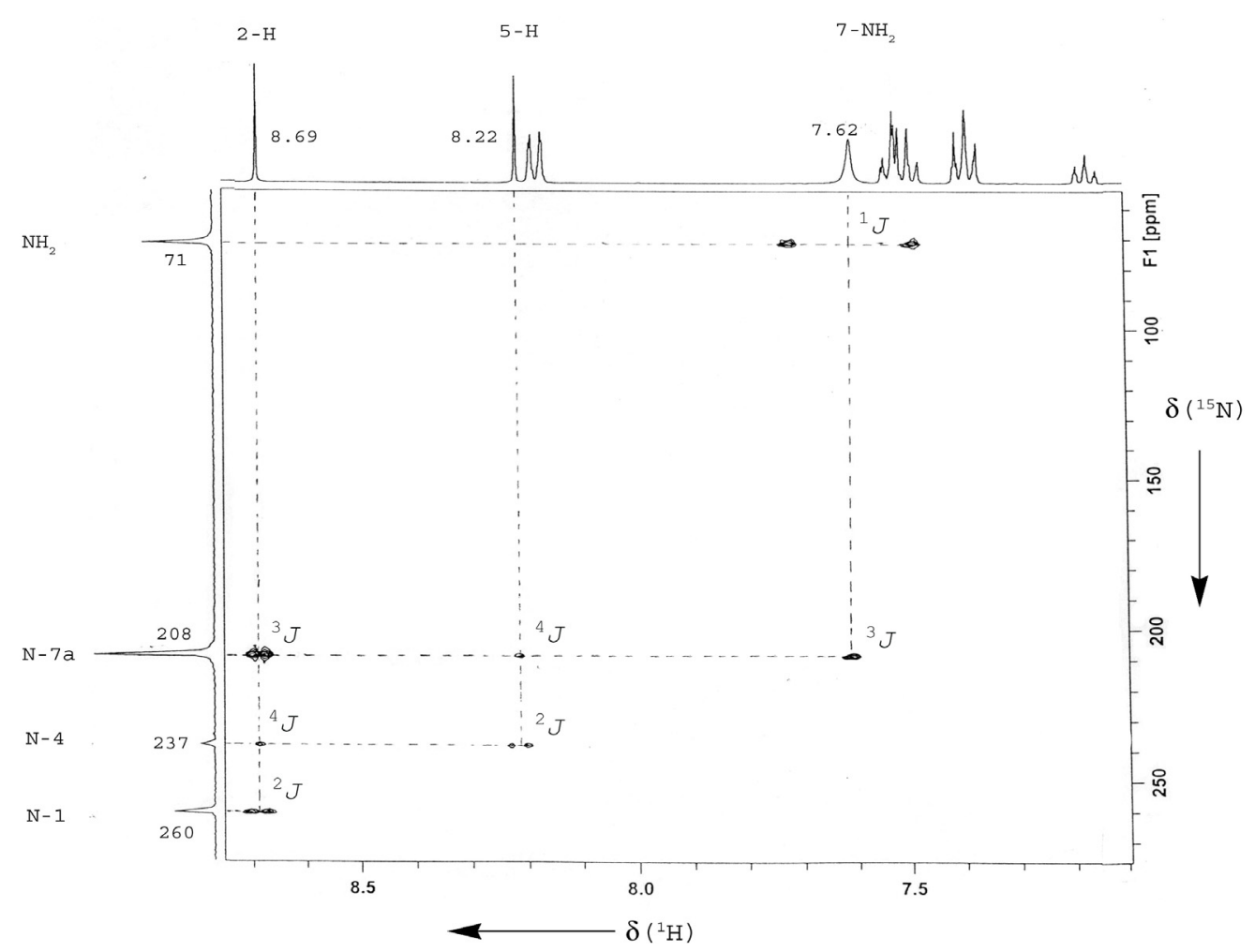

Figure 1. $\left({ }^{15} \mathrm{~N},{ }^{1} \mathrm{H}\right) \mathrm{HMBC}$ spectrum of 11 measured in $\mathrm{CD}_{3} \mathrm{SOCD}_{3}$. The $\delta\left({ }^{1} \mathrm{H}\right)$ values are related to TMS and the $\delta\left({ }^{15} \mathrm{~N}\right)$ values to $\mathrm{NH}_{3}$. 
Additionally to the $\left({ }^{15} \mathrm{~N},{ }^{1} \mathrm{H}\right) \mathrm{HMBC}$ spectrum, $\left({ }^{13} \mathrm{C},{ }^{1} \mathrm{H}\right) \mathrm{HSQC}$ and $\left({ }^{13} \mathrm{C},{ }^{1} \mathrm{H}\right) \mathrm{HMBC}$ spectra of 11 were measured. The three two-dimensional techniques permit a complete assignment of all ${ }^{1} \mathrm{H},{ }^{13} \mathrm{C}$ and ${ }^{15} \mathrm{~N}$ signals to certain nuclei of $\mathbf{1 1}$ (Figure 2).

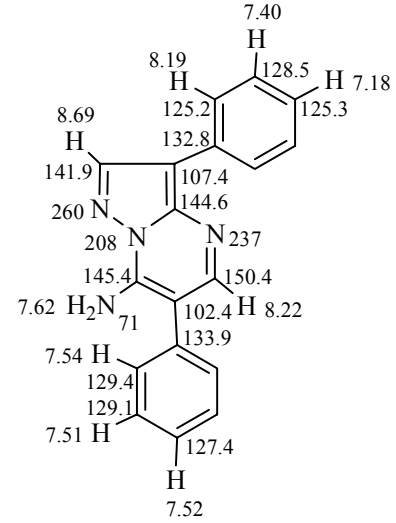

11

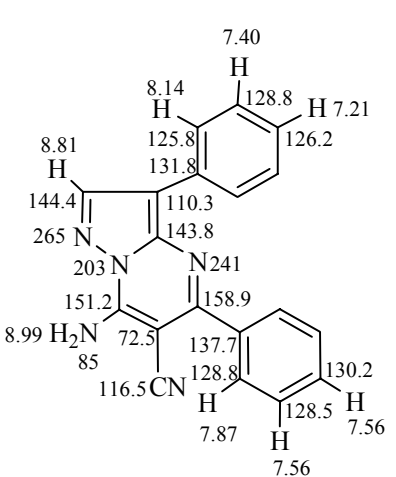

$10 a$

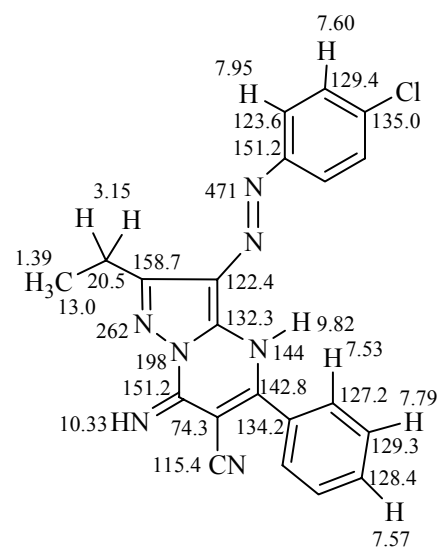

$10 b^{\prime}$

Figure 2. Assignment of the ${ }^{1} \mathrm{H},{ }^{13} \mathrm{C}$ and ${ }^{15} \mathrm{~N}$ NMR signals of the 3,6-diphenylpyrazolo[1,5a]pyrimidines 11, 10a and 10b'.

The structure of 10a corresponds to 11. Regioselective cyclization yields again a 7aminopyrazolo[1,5- $a$ ]pyrimidine. Figure 2 contains the complete assignment of all ${ }^{1} \mathrm{H},{ }^{13} \mathrm{C}$ and ${ }^{15} \mathrm{~N}$ NMR signals of 10a - based on the three 2D NMR techniques mentioned above. The $\delta\left({ }^{1} \mathrm{H}\right)$ and $\delta\left({ }^{15} \mathrm{~N}\right)$ values of $\mathbf{1 0 a}$ and $\mathbf{1 1}$ agree very well. A considerable difference exists only for the ${ }^{13} \mathrm{C}$ chemical shift of C-6. The cyano substituent shifts the $\delta$ value of this enamine carbon atom to higher field. When we applied the three 2D NMR techniques to $\mathbf{1 0 b}$, we recognized some new aspects. The ${ }^{1} \mathrm{H}$ NMR measurement in $\mathrm{CD}_{3} \mathrm{SOCD}_{3}$ revealed molecular dynamics. Compared to 11 and 10a, the NH signals are at lower field in 10b namely at $\delta=10.33$ and $9.82 \mathrm{ppm}$ and coalesce at $\delta=9.94$ on moderate warming to $40{ }^{\circ} \mathrm{C}$ (or enhancement of the $\mathrm{H}_{2} \mathrm{O}$ concentration). A rotational restriction of the amino group is unlikely. Therefore we have to consider a tautomeric equilibrium. Scheme 3 shows three possible tautomers $\mathbf{1 0 b}, \mathbf{1 0 b}$ and $\mathbf{1 0 b}$ ". The $\left({ }^{1} \mathrm{H}\right.$, ${ }^{15} \mathrm{~N}$ ) HMBC spectrum contains signals for $\mathrm{N}-1, \mathrm{~N}-4$ and $\mathrm{N}-7 \mathrm{a}$. Whereas the $\delta$ values of $\mathrm{N}-1$ and $\mathrm{N}-7$ a are very similar to the corresponding values of $\mathbf{1 1}$ and 10a, N-4 has now a $\delta$ value of 144 ppm, which is high-field shifted by almost $100 \mathrm{ppm}$ in comparision to $\mathbf{1 1}$ and 10a. This effect can be explained by a rehybridization of $\mathrm{N}-4$ from $\mathrm{sp}^{2}$ to $\mathrm{sp}^{3}$. The $\left({ }^{15} \mathrm{~N},{ }^{1} \mathrm{H}\right) \mathrm{HMBC}$ measurement at room temperature in $\mathrm{CD}_{3} \mathrm{SOCD}_{3}$ speaks for structure $\mathbf{1 0 b}^{\prime}$ as prevailing tautomer in solution in DMSO; for $10 b$ a $\delta$ value of about 240 ppm could be expected for $\mathrm{N}-4$. The ${ }^{15} \mathrm{~N}$ chemical shift of one of the nitrogen atoms in the azo group (Figure 2) can be assigned by the ${ }^{3} J\left({ }^{15} \mathrm{~N},{ }^{1} \mathrm{H}\right)$ coupling with the $o-\mathrm{H}$ of the benzene ring; its ${ }^{15} \mathrm{~N}$ chemical shift of 471 ppm precludes a hydrazono group present in $\mathbf{1 0 b}$ ". The other nitrogen atom of the azo group can not be seen in the $\left({ }^{15} \mathrm{~N},{ }^{1} \mathrm{H}\right) \mathrm{HMBC}$ spectrum because of a minor polarization transfer. The nitrogen atom of the 
amino/imino group can also not be seen - due to an exchange mechanism, which includes a $Z$ / $E$ (syn/anti) isomerism at this center. Figure 2 shows the assignment of the ${ }^{15} \mathrm{~N},{ }^{1} \mathrm{H}$ and ${ }^{13} \mathrm{C}$ NMR signals of $\mathbf{1 0 b}$ '.
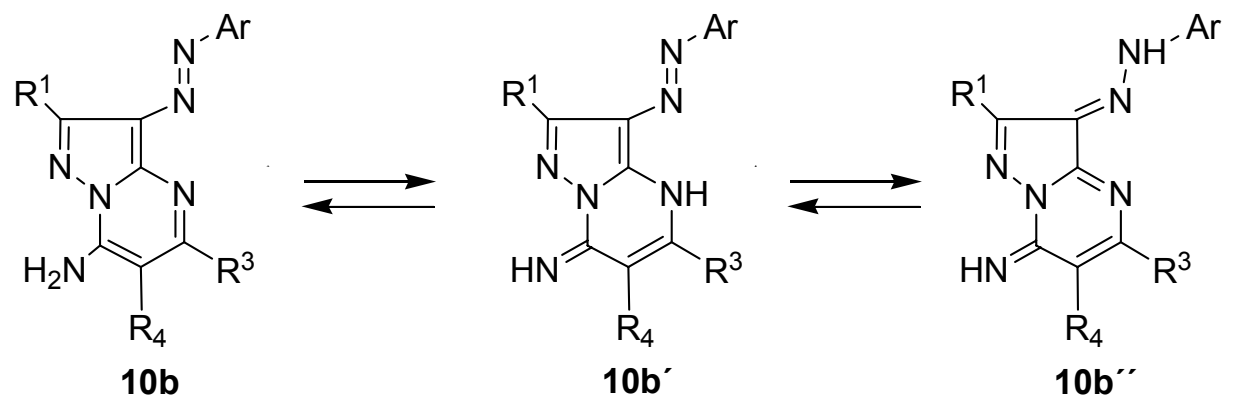

\section{Scheme 3}

We could obtain an X-ray crystal structure ${ }^{13}$ for the reaction product of $\mathbf{6}$ and $\mathbf{5 c}$. As clearly indicated (cf. figure 3 ) and contradicting previous believes ${ }^{10}$ the reaction product 10c is a 7aminopyrazolo[1,5-a]pyrimidine . The tautomerism of 10c in solution was not studied.

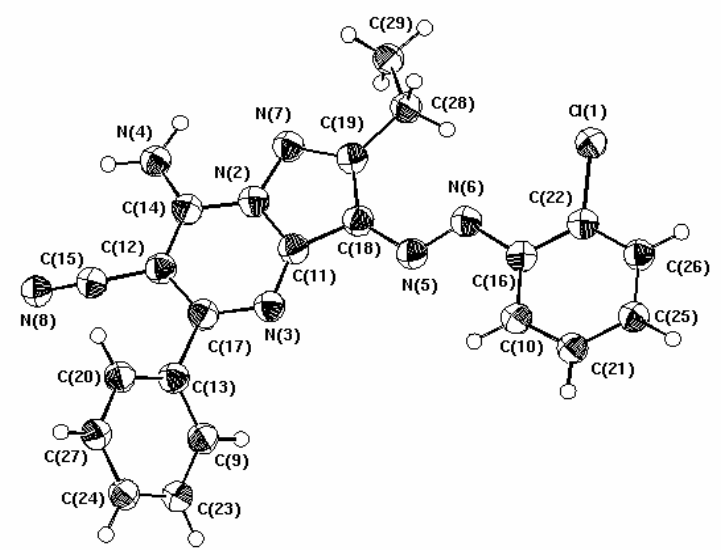

Figure 3. X-ray crystal structure for compound 10c. ${ }^{13}$

Similar to the behaviour of 5a-d toward $\mathbf{6}$, compound $\mathbf{5 b}$ also reacted with 3-(piperidin-1yl)acrylonitrile 13 to yield 14 (Scheme 4).

In conclusion $2 \mathrm{D}\left({ }^{15} \mathrm{~N},{ }^{1} \mathrm{H}\right) \mathrm{HMBC}$ measurements and X-ray crystal structure analyses can be readily utilized to establish the product structures of reacting aminoazoles with $\beta$-bifunctional reagents. All pyrazolo[1,5-a]pyrimidines discussed here bear the amino group on C-7. That proves a regioselective reaction of the 3-amino-1- $H$-pyrazoles 5 and $\alpha, \beta$-unsaturated nitriles $\mathbf{2 ,}, \mathbf{6}$, 13. 


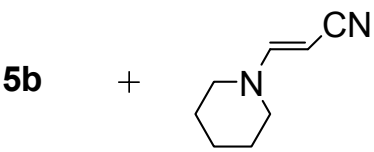

13<smiles>[R]c1nn2c(N)ccnc2c1[R]</smiles>

14

$$
\mathrm{R}^{1}=\mathrm{C}_{2} \mathrm{H}_{5}, \mathrm{R}^{2}=\mathrm{NNC}_{6} \mathrm{H}_{4}-p-\mathrm{Cl}
$$

\section{Scheme 4}

\section{Experimental Section}

General Procedures. All melting points are uncorrected. IR spectra were recorded in $\mathrm{KBr}$ with a Pye Unicam SP 1100 spectrophotometer. NMR spectra were recorded on a Bruker Avance DPX$300 \mathrm{MHz}$ spectrometer. Coupling constants $(J)$ are reported in $\mathrm{Hz}$, and chemical shifts are reported in parts per million $(\delta)$ relative to $\mathrm{DMSO}_{-} \mathrm{d}_{6}\left(2.49 \mathrm{ppm}\right.$ for ${ }^{1} \mathrm{H}$ and $39.5 \mathrm{ppm}$ for $\left.{ }^{13} \mathrm{C}\right)$. Two-dimensional NMR Bruker AMX 400 and Avance 600. Microwave irradiation was carried out using a commercial microwave oven (SGO $390 \mathrm{~W}$ ). Crystal structures were performed using Envaf Nonius 591 Kappa CCD single crystal diffraction. Ms spectra were recorded on a Fison Instrument VG ProSpec Q.

\section{3-Amino-4-phenyl-1H-pyrazole (5a) ${ }^{14}$}

One gram of 2 is placed in a $50 \mathrm{ml}$ conical then treated with hydrazine hydrate $(1 \mathrm{ml}: 80 \%)$ then with acetic acid $(2 \mathrm{ml})$ and DMF $(2 \mathrm{ml})$. The reaction mixture was then heated in a domestic microwave oven at full power for 3 minutes. The resulting product was then triturated with water and the so formed solid was collected by filtration then crystallized from ethanol, (yield 67\%, 0.5

$\mathrm{g}$ of 5a. The product was found identical (identity was made by mp $176-177{ }^{\circ} \mathrm{C}$, lit. ${ }^{14} \mathrm{mp} 176{ }^{\circ} \mathrm{C}$ and mixed $\mathrm{mp}$ 175-176) with product obtained following literature procedure; MS (EI) $\mathrm{m} / \mathrm{z} 159$ $\left(\mathrm{M}^{+}, 100 \%\right)$.

General procedure for the preparation of 4-(arylazo)-5-ethyl-1 $H$-pyrazol-3-ylamine (5b-d) A mixture of 4a-d $(10 \mathrm{mmol})$ in $20 \mathrm{ml} \mathrm{EtOH}$, and hydrazine hydrate $80 \%(0.75 \mathrm{~g}, 15 \mathrm{mmol})$ was heated under reflux for $2 \mathrm{~h}$. The reaction mixture was then poured into water, filtered off and recrystallized from ethanol.

4-((4-Chlorophenyl)diazenyl)-5-ethyl-1H-pyrazol-3-amine (5b). This compound was obtained as yellow crystals, (yield 74\%, $1.84 \mathrm{~g}$ ) mp 181-182 ${ }^{\circ} \mathrm{C}$; IR (KBr): $v_{\max } 3414$ (s), 3298 (s), 3167 (w) $\mathrm{cm}^{-1}\left(\mathrm{NH}_{2} \& \mathrm{NH}\right) ;{ }^{1} \mathrm{H}$ NMR $\left(300 \mathrm{MHz}, \mathrm{DMSO}-d_{6}\right): \delta 1.30\left(\mathrm{t}, 3 \mathrm{H}, J=7.5 \mathrm{~Hz}, \mathrm{CH}_{3}\right), 2.81$ (q, $2 \mathrm{H}, J=7.5 \mathrm{~Hz}, \mathrm{CH}_{2}$ ), 6.56 (brs, 2H, NH ), 7.44 (d, 2H, $\left.J=8.6 \mathrm{~Hz}, \mathrm{Ar}-\mathrm{H}\right), 7.68$ (d, 2H, $J=8.6$ $\mathrm{Hz}, \mathrm{Ar}-\mathrm{H}), 11.77 \mathrm{ppm}$ (brs, 1H, NH); ${ }^{13} \mathrm{C}$ NMR (75 MHz, DMSO-d 6 ): $\delta 152.8(\mathrm{C}), 132.6(\mathrm{C})$, $132.5(\mathrm{C}), 129.8(\mathrm{CH}), 129.7(\mathrm{CH}), 123.1(\mathrm{C}), 97.9(\mathrm{C}), 19.7\left(\mathrm{CH}_{2}\right), 14.1\left(\mathrm{CH}_{3}\right)$ ppm; MS (EI) 
m/z $249\left(\mathrm{M}^{+}, 100 \%\right)$. Anal. (HRMS) $\mathrm{C}_{11} \mathrm{H}_{12} \mathrm{ClN}_{5}$; Mass Cal: 249.078123; Mass found: 249.077586; Anal. calcd: C, 52.91; H, 4.84; N, 28.05. Found: C, 52.83; H, 4.69; N, 27.87.

4-((2-Chlorophenyl)diazenyl)-5-ethyl-1H-pyrazol-3-amine (5c). This compound was obtained as yellow crystals, (yield $69 \%, 1.72 \mathrm{~g}$ ) mp 126-128 ${ }^{\circ} \mathrm{C}$; IR (KBr): $v_{\max } 3437$ (s br), 3309 (s) cm ${ }^{-1}$ $\left(\mathrm{NH}_{2} \& \mathrm{NH}\right) ;{ }^{1} \mathrm{H}$ NMR $\left(300 \mathrm{MHz}, \mathrm{DMSO}-d_{6}\right): \delta 1.20\left(\mathrm{t}, 3 \mathrm{H}, J=7.5 \mathrm{~Hz}, \mathrm{CH}_{3}\right), 2.72(\mathrm{q}, 2 \mathrm{H}, J=$ $7.5 \mathrm{~Hz}, \mathrm{CH}_{2}$ ), 6.32 ppm (brs, 1H, NH), 7.2-7.43 (m, 2H, Ar-H), 7.60 (d, 1H, J=7.3 Hz, Ar-H), $7.78(\mathrm{~d}, 1 \mathrm{H}, J=8.1 \mathrm{~Hz}, \mathrm{Ar}-\mathrm{H}), 11.95 \mathrm{ppm}$ (brs, 2H, NH) ); ${ }^{13} \mathrm{C}$ NMR (75 MHz, DMSO-d $)$ : $\delta$ $149.3(\mathrm{C}), 131.6(\mathrm{C}), 130.9(\mathrm{CH}), 129.7(\mathrm{C}), 129.5(\mathrm{CH}), 129.4(\mathrm{C}), 128.7(\mathrm{CH}), 117.1(\mathrm{CH})$, 98.1 (C), $19.8\left(\mathrm{CH}_{2}\right), 14.0\left(\mathrm{CH}_{3}\right)$ ppm; MS (EI) m/z $249\left(\mathrm{M}^{+}, 71 \%\right) 214$ (81) 138 (100). Anal. (HRMS) $\mathrm{C}_{11} \mathrm{H}_{12} \mathrm{ClN}_{5}$ Mass Cal: 249.078123; Mass found: 249.078095; Anal. calcd: C, 52.91; H, 4.84; N, 28.05. Found: 52.77; H, 4.98; N, 27.93.

5-Ethyl-4-(p-tolyldiazenyl)-1H-pyrazol-3-amine (5d). This compound was obtained as yellow crystals, (yield 71\%, $1.63 \mathrm{~g}$ ) mp 150-152 ${ }^{\circ} \mathrm{C}$; IR (KBr): $v_{\max } 3418$ (s br), 3305 (s br) cm ${ }^{-1}\left(\mathrm{NH}_{2}\right.$ \& $\mathrm{NH}) ;{ }^{1} \mathrm{H}$ NMR (300 MHz, DMSO- $\left.d_{6}\right): \delta 1.30\left(\mathrm{t}, 3 \mathrm{H}, J=7.5 \mathrm{~Hz}, \mathrm{CH}_{3}\right), 2.40\left(\mathrm{~s}, 3 \mathrm{H}, \mathrm{CH}_{3}\right), 2.75$ (q, $2 \mathrm{H}, J=7.5 \mathrm{~Hz}, \mathrm{CH}_{2}$ ), 6.56 (brs, 2H, $\left.\mathrm{NH}_{2}\right), 7.44$ (d, 2H, $\left.J=8.1 \mathrm{~Hz}, \mathrm{Ar}-\mathrm{H}\right), 7.68$ (d, 2H, $J=8.1$ $\mathrm{Hz}, \mathrm{Ar}-\mathrm{H}), 11.77 \mathrm{ppm}$ (brs, 1H, NH); ${ }^{13} \mathrm{C}$ NMR (75 MHz, DMSO-d 6 ): $\delta 151.9$ (C), $138.3(\mathrm{C})$, $130.7(\mathrm{C}), 130.4(\mathrm{CH}), 123.3(\mathrm{CH}), 121.5(\mathrm{C}), 98.1(\mathrm{C}), 21.6\left(\mathrm{CH}_{3}\right), 19.5\left(\mathrm{CH}_{2}\right), 14.2\left(\mathrm{CH}_{3}\right)$ ppm; MS (EI) m/z 229 (M $\mathrm{M}^{+}, 100 \%$ ). Anal. (HRMS) $\mathrm{C}_{12} \mathrm{H}_{15} \mathrm{~N}_{5}$ Mass cal: 229.132746; Mass found: 229.133137; Anal. calcd: C, 62.86; H, 6.59; N, 30.54. Found: C, 62.98; H, 6.44; N, 30.41. 7-Amino-3,5-diphenyl-pyrazolo[1,5-a]pyrimidine-6-carbonitrile (10a). A solution of 5a (1 $\mathrm{mmol})$ and bezylidenemalononitrile $(1 \mathrm{mmol})$ in pyridine $(10 \mathrm{ml})$ was refluxed for $6 \mathrm{~h}$, then left to cool to room temperature. The reaction mixture was then poured onto water and solid obtained after neutralisation with dilute hydrochloric acid was collected by filtration and crystallized from dioxan. This compound was obtained as buff crystals, (yield $74 \%, 0.23 \mathrm{~g}$ ) mp 214-215 ${ }^{\circ} \mathrm{C}$; IR $(\mathrm{KBr}): v_{\max } 3410(\mathrm{w}), 3320(\mathrm{w})\left(\mathrm{NH}_{2}\right), 2220(\mathrm{~s}) \mathrm{cm}^{-1}(\mathrm{CN})$; NMR (400 MHz, DMSO- $\left.d_{6}\right): \delta 7.17-$ $7.25(\mathrm{~m}, 1 \mathrm{H}), 7.38-7.42(\mathrm{~m}, 2 \mathrm{H}), 7.54-7.58(\mathrm{~m}, 3 \mathrm{H}), 7.85-7.90(\mathrm{~m}, 2 \mathrm{H}), 8.10-8.18(\mathrm{~m}, 2 \mathrm{H}), 8.81$ $(\mathrm{s}, 1 \mathrm{H}), 8.99 \mathrm{ppm}$ (brs, 2H); ${ }^{13} \mathrm{C}$ NMR (100 MHz, DMSO-d $)$ : $\delta 158.9(\mathrm{C}), 151.2(\mathrm{C}), 144.4$ $(\mathrm{CH}), 143.8(\mathrm{C}), 137.7(\mathrm{C}), 131.8(\mathrm{C}), 130.2(\mathrm{CH}), 128.8(\mathrm{CH}), 128.8(\mathrm{CH}), 128.5(\mathrm{CH}), 126.2$ $(\mathrm{CH}), 125.8(\mathrm{CH}), 116.5(\mathrm{C}), 110.3(\mathrm{C}), 72.5(\mathrm{C}) \mathrm{ppm}$; MS (EI) m/z $311\left(\mathrm{M}^{+}, 100 \%\right)$. Anal. Calcd $\left(\mathrm{C}_{19} \mathrm{H}_{13} \mathrm{~N}_{5}\right)$ : C, 73.30; H, 4.21; N, 22.49. Found: C, 73.42; H, 4.11; N, 22.35.

General procedure for the preparation of 7-Amino-3-(arylazo)-5-phenyl-pyrazolo[1,5a]pyrimidine-6-carbonitrile (10b-d)

A mixture of 5b-d $(1 \mathrm{mmol})$ in pyridine $(10 \mathrm{ml})$, and benzylidenemalononitrile $(0.154 \mathrm{~g}, 1$ mmol) was refluxed for $6 \mathrm{~h}$. the reaction mixture was then poured into ice water, and neutralized by $\mathrm{HCl}$, filtered off and recrystallized from ethanol.

7-Amino-3-(4'-chlorophenylazo)-2-ethyl-5-phenyl-pyrazolo[1,5-a]pyrimidine-6-carbonitrile (10b).This compound was obtained as red crystals, (yield $65 \%, 0.26 \mathrm{~g}$ ) mp 232-234 ${ }^{\circ} \mathrm{C}$; IR $(\mathrm{KBr}): v_{\max } 3297(\mathrm{~s}), 3224(\mathrm{~s})\left(\mathrm{NH}_{2}\right), 2214(\mathrm{~s}) \mathrm{cm}^{-1}(\mathrm{CN}) ;{ }^{1} \mathrm{H}$ NMR (300 MHz, DMSO- $\left.d_{6}\right): \delta$ 1.39 (t, 3H, $\left.J=7.4 \mathrm{~Hz}, \mathrm{CH}_{3}\right), 3.15$ (q, 2H, $J=7.4 \mathrm{~Hz}, \mathrm{CH}_{2}$ ), 7.50-7.61 (m, 5H, Ar-H and ph-H), 7.77-7.80 (m, 2H, ph-H), 7.86-7.90 (m, 2H, Ar-H), 9.12 ppm (brs, $\left.2 \mathrm{H}, \mathrm{NH}_{2}\right)$; ${ }^{13} \mathrm{C}$ NMR $(100$ 
MHz, DMSO- $\left.d_{6}\right): \delta 158.7(\mathrm{C}), 151.2(\mathrm{C}), 151.2(\mathrm{C}), 142.8(\mathrm{C}), 135.0(\mathrm{C}), 134.2(\mathrm{C}), 132.3(\mathrm{C})$, $129.4(\mathrm{CH}), 129.3(\mathrm{CH}), 128.4(\mathrm{CH}), 127.2(\mathrm{CH}), 123.6(\mathrm{CH}), 122.4(\mathrm{C}), 115.4(\mathrm{C}), 74.3(\mathrm{C})$, $20.5\left(\mathrm{CH}_{2}\right), 13.0\left(\mathrm{CH}_{3}\right)$ ppm; MS (EI) m/z $401\left(\mathrm{M}^{+}, 100 \%\right)$. Anal. (HRMS) $\mathrm{C}_{21} \mathrm{H}_{16} \mathrm{ClN}_{7}$ Calcd. Mass: 401.115571 Mass found: 401.115192; Anal. calcd: C, 62.77; H, 4.01; N, 24.40. Found: C, $62.71 ; \mathrm{H}, 4.10 ; \mathrm{N}, 24.32$.

7-Amino-3-(2'-chlorophenylazo)-2-ethyl-5-phenyl-pyrazolo[1,5-a]pyrimidine-6-carbonitrile

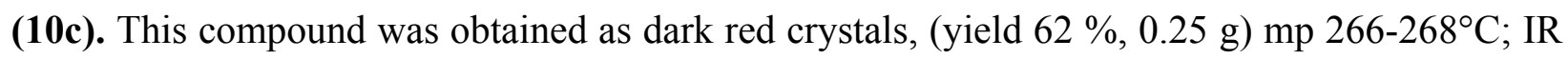
$(\mathrm{KBr}): v_{\max } 3437(\mathrm{~s}), 3292(\mathrm{~s})\left(\mathrm{NH}_{2}\right), 2222(\mathrm{~s}) \mathrm{cm}^{-1}(\mathrm{CN}) ;{ }^{1} \mathrm{H}$ NMR (300 MHz, DMSO- $\left.d_{6}\right): \delta$ 1.32 (t, 3H, $J=7.5 \mathrm{~Hz}, \mathrm{CH}_{3}$ ), 3.13 (q, 2H, $J=7.5 \mathrm{~Hz}, \mathrm{CH}_{2}$ ), 7.41-7.48 (m, 2H, Ar-H), 7.50-7.76 (m, 5H, Ar-H), 7.83-7.92 (m, 2H, Ar-H), 9.12 ppm (brs, 2H, NH $) ;{ }^{13} \mathrm{C}$ NMR (75 MHz, DMSO$\left.d_{6}\right): \delta 162.9(\mathrm{C}), 154.2(\mathrm{C}), 151.5(\mathrm{C}), 149.8(\mathrm{C}), 146.9(\mathrm{C}), 138.0(\mathrm{C}), 133.7(\mathrm{C}), 132.0(\mathrm{CH})$, $131.7(\mathrm{CH}), 131.4(\mathrm{CH}), 129.6(\mathrm{CH}), 129.2(\mathrm{CH}), 128.7(\mathrm{CH}), 127.6(\mathrm{C}), 117.6(\mathrm{CH}), 116.7(\mathrm{C})$, $76.4(\mathrm{C}), 23.5\left(\mathrm{CH}_{2}\right), 12.9\left(\mathrm{CH}_{3}\right)$ ppm; X-ray crystal structure are shown cf. figure 3; MS (EI) m/z $401\left(\mathrm{M}^{+}, 95 \%\right) 366$ (39) 290 (100). Anal. (HRMS) $\mathrm{C}_{21} \mathrm{H}_{16} \mathrm{ClN}_{7}$ Calcd. Mass: 401.115571; Mass found: 401.114757; Anal. calcd: C, 62.77; H, 4.01; N, 24.40. Found: C, 62.63; H, 3.93; N, 24.30 .

7-Amino-2-ethyl-5-phenyl-3-p-tolylazo-pyrazolo[1,5-a]pyrimidine-6-carbonitrile (10d). This compound was obtained as red crystals, (yield $68 \%, 0.26 \mathrm{~g}$ ) $\mathrm{mp} 263-265{ }^{\circ} \mathrm{C}$; IR (KBr): $v_{\max }$ 3425 (s), $3298(\mathrm{~s})\left(\mathrm{NH}_{2}\right), 2218(\mathrm{~s}) \mathrm{cm}^{-1}(\mathrm{CN}) ;{ }^{1} \mathrm{H}$ NMR $\left(300 \mathrm{MHz}, \mathrm{DMSO}-d_{6}\right): \delta 1.30(\mathrm{t}, 3 \mathrm{H}, J=$ $\left.7.4 \mathrm{~Hz}, \mathrm{CH}_{3}\right), 2.35$ (s, 3H, $\left.\mathrm{CH}_{3}\right), 3.20\left(\mathrm{q}, 2 \mathrm{H}, J=7.4 \mathrm{~Hz}, \mathrm{CH}_{2}\right), 7.35$ (d, 2H, J=8.2 Hz, Ar-H), 7.50-7.58 (m, 3H, ph-H), 7.66 (d, 2H, $J=8.2 \mathrm{~Hz}, \mathrm{Ar}-\mathrm{H}), 7.82-7.89$ (m, 2H, ph-H), 9.10 ppm (brs, $\left.2 \mathrm{H}, \mathrm{NH}_{2}\right) ;{ }^{13} \mathrm{C}$ NMR (75 MHz, DMSO- $\left.d_{6}\right): \delta 162.4(\mathrm{C}), 154.7(\mathrm{C}), 152.1(\mathrm{C}), 151.4(\mathrm{C})$, $145.0(\mathrm{C}), 140.6(\mathrm{C}), 138.1(\mathrm{C}), 131.2(\mathrm{CH}), 130.6(\mathrm{CH}), 129.6(\mathrm{CH}), 129.2(\mathrm{CH}), 126.6(\mathrm{C})$, $122.3(\mathrm{CH}), 116.0(\mathrm{C}), 75.7(\mathrm{C}), 23.8\left(\mathrm{CH}_{2}\right), 21.8\left(\mathrm{CH}_{3}\right), 13.2\left(\mathrm{CH}_{3}\right) \mathrm{ppm} ; \mathrm{MS}(\mathrm{EI}) \mathrm{m} / \mathrm{z} 381\left(\mathrm{M}^{+}\right.$, 100\%) 290 (53). Anal. (HRMS) $\mathrm{C}_{22} \mathrm{H}_{19} \mathrm{~N}_{7}$ Calcd. Mass: 381.170794; Mass found: 381.171031; Anal. calcd: C, 69.27; H, 5.02; N, 25.70. Found: C, 69.12; H, 5.15; N, 25.56.

7-Amino-3,6-diphenylpyrazolo[1,5-a]pyrimidine (11). A solution of 5a (1 mmol) and 2 (1 $\mathrm{mmol})$ in pyridine $(10 \mathrm{ml})$ was refluxed for $5 \mathrm{~h}$ then left to cool to room temperature, poured onto water and neutralised by diluted hydrochloric acid. The solid product, so formed was collected by filtration and crystallized from dioxan, (yield $70 \%, 0.15 \mathrm{~g}$ ) $\mathrm{mp} 221-223{ }^{\circ} \mathrm{C}$ of the product that was found identical in every respect with the literature product ${ }^{15}$, lit. mp $222-224$ ${ }^{\circ} \mathrm{C}$. All NMR data are shown on figure 1 and 2 in discussions; MS (EI) m/z $286\left(\mathrm{M}^{+}, 100 \%\right)$.

3-(4'-Chlorophenylazo)-2-ethyl-pyrazolo[1,5-a]pyrimidin-7-ylamine (14). A mixture of 5b $(0.249 \mathrm{~g}, 1 \mathrm{mmol})$ in pyridine $(10 \mathrm{ml})$, and 3-piperidin-1-yl-acrylonitrile $(0.136 \mathrm{~g}, 1 \mathrm{mmol})$ was refluxed for $6 \mathrm{~h}$ the reaction mixture was then poured into ice water, and neutralize by $\mathrm{HCl}$, filtered off and recrystallized from ethanol to give orange crystals, (yield 67\%, $0.2 \mathrm{~g}$ ) $\mathrm{mp} 233$ $235{ }^{\circ} \mathrm{C}$; IR (KBr): $v_{\max } 3392$ (w br), 3234 (w br) $\mathrm{cm}^{-1}\left(\mathrm{NH}_{2}\right) ;{ }^{1} \mathrm{H}$ NMR (300 MHz, DMSO-d $): \delta$ 1.39 (t, 3H, $\left.J=7.2 \mathrm{~Hz}, \mathrm{CH}_{3}\right), 3.14\left(\mathrm{q}, 2 \mathrm{H}, J=7.2 \mathrm{~Hz}, \mathrm{CH}_{2}\right), 6.59$ (d, 1H, $\left.J=6.4 \mathrm{~Hz}, \mathrm{CH}\right), 7.60$ $(\mathrm{d}, 2 \mathrm{H}, J=8.7 \mathrm{~Hz}, \mathrm{Ar}-\mathrm{H}), 7.95(\mathrm{~d}, 2 \mathrm{H}, J=8.7 \mathrm{~Hz}, \mathrm{Ar}-\mathrm{H}), 8.29(\mathrm{~d}, 2 \mathrm{H}, J=6.4 \mathrm{~Hz}, \mathrm{CH}), 9.57$ ppm (brs, 2H, $\left.\mathrm{NH}_{2}\right) ;{ }^{13} \mathrm{C}$ NMR (75 MHz, DMSO- $\left.d_{6}\right): \delta 159.3(\mathrm{C}), 152.0(\mathrm{C}), 151.8(\mathrm{C}), 144.0$ 
$(\mathrm{CH}), 135.7(\mathrm{C}), 133.6(\mathrm{C}), 130.2(\mathrm{CH}), 124.4(\mathrm{CH}), 123.3(\mathrm{C}), 94.9(\mathrm{CH}), 21.3\left(\mathrm{CH}_{2}\right), 13.8$ $\left(\mathrm{CH}_{3}\right)$ ppm; MS (EI) m/z $300\left(\mathrm{M}^{+}, 99 \%\right) 189(100 \%)$. Anal. (HRMS) $\mathrm{C}_{14} \mathrm{H}_{13} \mathrm{ClN}_{6}$ Calcd. Mass: 300.089022; Mass found: 300.089073; Anal. calcd: C, 55.91; H, 4.36; N, 27.94. Found: C, 55.78; H, 4.44; N, 27.76.

\section{References}

1. Shiota, T.; Yamamori, T.; Sakai, K.; Kiyokawa, M.; Honma, T.; Ogawa, M.; Hayashi, K.; Ishizuka, N.; Matsumura, K.; Hara, M.; Fujimoto, M.; Kawabata, T.; Nakajima, S. Chem. Pharm. Bull. 1999, 47, 928.

2. Li, M.; Guo, W.; Wen, L.; Yang, H. Youji Huaxue 2005, 25, 1230.

3. Clasby, M. C.; Chackalamannil, S.; Neustadt, B. R.; Gao, X. U.S. Pat. Appl. Publ. 79 pp, 2006.

4. Bold, G.; Floersheimer, A.; Furet, P.; Imbach, P.; Masuya, K.; Schoepfer, J. G. U.S. Pat. Appl. Publ. 60 pp 2005.

5. Selleri, S.; Bruni, F.; Costagli, C.; Costanzo, A.; Guerrini, G.; Ciciani, G.; Costa, B.; Martini, C. Bioorg. \& Med. Chem. 1999, 7, 2705.

6. Foster, A, C.; Pelleymounter, M. A.; Cullen, M. J.; Lewis, D.; Joppa, M.; Chen, T.; Bozigian, H. P.; Gross, R. S.; Gogas, K. R. J. Pharmacol. Exp. Ther. 2004, 311, 547.

7. Wang, S. Q.; Fang, L.; Liu, X. J.; Zhao, K. Chinese Chem. Lett. 2004, 15, 885.

8. Mustazza, C.; Del Giudice, M. R.; Borioni, A.; Gatta, F. J. Heterocyclic Chem. 2001, 38, 1119.

9. Elnagdi, M. H.; Elmoghayar, M. R. H.; Elgemeie, G. E. H. Advances in Heterocyclic Chem. 1987, 41, 319.

10. Elfahham, H. A.; Abdel-Galil, F. M.; Ibraheim, Y. R.; Elnagdi, M. H. J. Heterocyclic Chem. 1983, 20, 667.

11. Al-Enezi, A.; Al-Saleh, B.; Elnagdi, M. H. J. Chem. Res., S 1997, 4.

12. Gozlan, S. A. S.; Abdelhamid, I. A.; Gaber, H.; Elnagdi, M. H. J. Chem. Res. 2004, 789.

13. Cambridge Crystallographic Data Centre and allocated the deposition number, CCDC 614742.

14. Ege G.; Franz H. J. Heterocyclic Chem. 1982, 19, 1267.

15. Alcade, E.; Mendoza, J.; Garcia-Marquina, J. M.; Almera, C.; Elguero, J. J. Heterocyclic Chem., 1974, 11, 423. 